\title{
Antimicrobial Resistance Pattern In Escherichia Coli Isolated From Children Admitted At Selected Health Care Facilities In Moshi Municipality, Tanzania
}

Mzelifa Daud ( $\nabla$ mzelifadaud@hotmail.com )

Kolandoto College https://orcid.org/0000-0003-2415-3792

Balthazar Nyombi

Kilimanjaro Christian Medical University College

\section{Research note}

Keywords: Antimicrobial resistance, Escherichia coli, children.

Posted Date: June 28th, 2019

DOl: https://doi.org/10.21203/rs.2.10704/v1

License: (c) (i) This work is licensed under a Creative Commons Attribution 4.0 International License.

Read Full License

Version of Record: A version of this preprint was published at South Asian Journal of Research in Microbiology on June 9th, 2020. See the published version at https://doi.org/10.9734/sajrm/2020/v6i330153. 


\section{Abstract}

Background The emergence of antimicrobial resistance is a great challenge in preventing, controlling and treatment Gram-negative bacteria and has become a significant public health threat worldwide. The study aimed at determination of the prevalence and pattern of antimicrobial resistance in Escherichia coli isolated in children admitted at health facilities in Moshi municipality, Tanzania. Methodology A descriptive cross sectional study was carried out on archived rectal swabs collected from 282 children aged 3 to 168 months admitted at Kilimanjaro Christian Medical Centre (KCMC), Mawenzi Regional Referral Hospital and Pasua health centre in Moshi municipality. Rectal swabs were cultured on MacConkey agar media. The isolated Escherichia coli was identified and antimicrobial susceptibility test was done by using Kirby-Bauer disc diffusion method on Muller Hinton agar media. Results Escherichia coli were isolated in all 282 cultured archived rectal swabs and all isolates were susceptible to amikacin and meropenem. ESBL producers were $13.1 \%$ and $86.9 \%$ were non-ESBLs. Conclusion High resistance rate were observed on ampicillin, ceftriaxone, ceftazidime, cefuroxime, trimethoprim and ciprofloxacin among ESBL producing Escherichia coli. Among non ESBL producing Escherichia coli high resistance rate were observed on ampicillin and trimethoprim; however all isolates were sensitive to amikacin and meropenem.

\section{Introduction}

Antimicrobial resistance (AMR) development among Gram negative bacteria has become a significant public health threat worldwide. Currently there are fewer antimicrobials available for treatment and prevention of some of bacterial infections (1).Antimicrobial resistance development in some of bacterial strains undermines empirical treatment regimens, thereby limiting choice of appropriate antibiotic (2).As this problem continues to grow, epidemiological surveillance is warranted to generate data that is reliable to understand the distribution of AMR among healthcare settings and geographical regions (3).

AMR among Gram-negative bacteria poses a challenge in treating and controlling bacterial infectious diseases worldwide $(4,5)$. The currently available information on the prevalence and patterns of AMR in different geographical regions has demonstrated that it is on rise $(6,7,8)$. The recommended and available antimicrobials have shown to be losing their ability to kill most of the infectious bacteria by developing resistance and becoming difficult to treat patients (9). Some bacteria pose ability to produce enzymes such as Extended Spectrum $\beta$-Lactamase (ESBL), which is responsible for the resistance to cephalosporin and penicillin (10). The increase in circulating resistant strains of infectious bacteria make it difficult for the use of current available and recommended antimicrobial drugs (11).

Resistance of Gram negative bacteria to the recommended and available antimicrobial drugs is a growing public health problem in different locations worldwide leading to increased morbidity and mortality to patients (12). 
Surveillance report has shown that 500000 people suffer from antimicrobial resistance worldwide (13). The report on AMR in Africa revealed varying degree of AMR on the tested antimicrobials like amoxicillin $72.9 \%$, trimethoprim/sulfamethoxazole $75 \%$, gentamicin $22.1 \%$ and ceftriaxone $17.2 \%$ (14). The study on AMR pattern at tertiary hospital in Tanzania showed that resistance of Klebsiella pneumoniae and Escherichia coli isolates to third generation cephalosporin were $38.5 \%$ and $29.3 \%$ respectively (15). Although AMR has been reported worldwide, there is limited information on Escherichia coli isolates in children admitted at health facilities in Moshi Municipality, Tanzania. Therefore, this study aimed to determine the Escherichia coli carriages and patterns of antibiotic resistance in children admitted at selected health facilities in Moshi Municipality, Tanzania.

\section{Methods}

\section{Study design and setting}

This was a descriptive cross-sectional facility-based study conducted in Moshi Municipality from October 2017 to April 2018 . The archived samples which were preserved at $-80^{\circ} \mathrm{C}$ in teaching laboratory of Kilimanjaro Christian Medical University College (KCMUCo) were obtained from pediatric wards of three purposively sampled health facilities in Moshi municipality, Northern Tanzania; Kilimanjaro Christian Medical centre (KCMC) is located in the Northern part of Tanzania, $\mathrm{KCMC}$ is a zonal referral hospital, Mawenzi Regional Referral Hospital is located at the centre of Moshi Town, Mawenzi Regional Referral Hospital is a referral hospital for Kilimanjaro region and Pasua health centre which is a primary health care facility in Moshi municipality. All identified Escherichia coli isolates were included into the study.

\section{Bacterial isolates}

Archived rectal swabs collected from all enrolled children aged 3 to 168 months admitted at Kilimanjaro Medical Centre, Mawenzi Regional Referral hospital and Pasua Health Centre was cultured for bacterial growth at the teaching laboratory of Kilimanjaro KCMUCo in Moshi. Rectal swabs were first inoculated on MacConkey gar (MCA) plates and incubated at $37^{\circ} \mathrm{C}$ for 16 hours. Grown colonies were identified by using Kligler Iron Agar (KIA), Sulfide Indole and Motility (SIM), citrate utilization as well as Gram stain.

\section{Antimicrobial susceptibility testing}

Isolated microorganism was emulsified into the normal saline; turbidity was compared with 0.5 MacFarland turbidity standards. Antimicrobial susceptibility testing (AST) was done by Kirby-Bauer disc diffusion method using Muller Hinton agar (MHA) which was then incubated for 16 hours at $37^{\circ} \mathrm{C}$. Antimicrobial susceptibility testing was performed on selected antibiotics recommended by Tanzania standard Treatment Guidelines (Ministry of Health, 2017). Other antimicrobials are available in the pharmacies around Moshi Municipality, were also included in the study. The antimicrobial drugs tested were: ampicillin $(10 \mu \mathrm{g})$, chloramphenicol $(30 \mu \mathrm{g})$, amikacin $(30 \mu \mathrm{g})$, ciprofloxacin $(5 \mu \mathrm{g})$, trimethoprim 
$(5 \mu \mathrm{g})$, gentamicin $(10 \mu \mathrm{g})$, piperacillin-tazobactam $(100 / 10 \mu \mathrm{g})$, amoxicillin-clavunic $(20 / 10 \mu \mathrm{g})$, ceftazidime $(30 \mu \mathrm{g})$, meropenem $(10 \mu \mathrm{g})$, ceftriaxone $(30 \mu \mathrm{g})$ and cefuroxime $(30 \mu \mathrm{g})$.

The zone of bacterial growth inhibition by the antibiotics was measured in millimeter $(\mathrm{mm})$ according to Clinical Laboratory Standard Institute (CLSI) guideline of 2013. Escherichia coli ATCC 25922 was used as quality control strain. The following zone of inhibition was considered as resistant; $\leq 13 \mathrm{~mm}$ for ampicillin and amoxicillin/clavunic acid, $\leq 12 \mathrm{~mm}$ for gentamicin and chloramphenicol, $\leq 17 \mathrm{~mm}$ for pipercillintazobactam and ceftazidime, $\leq 19 \mathrm{~mm}$ for ceftriaxone and meropenem, $\leq 20 \mathrm{~mm}$ for ciprofloxacin, $\leq$ $14 \mathrm{~mm}$ for amikacin, $\leq 10 \mathrm{~mm}$ for trimethoprim and $\leq 14 \mathrm{~mm}$ for cefuroxime.

\section{Detection of ESBL}

All isolates resistant to cefuroxime were tested for ESBL production whereby ceftazidime was set $2 \mathrm{~cm}$ away from amoxicillin/clavunic acid and synergism effect was determined. A test was considered as positive when a decreased susceptibility to ceftazidime was combined with a clear-cut enhancement of the inhibition zone of ceftazidime in front of amoxicillin/clavunic disc, often resulting in a characteristic shape-zone referred to as champagne-cork or keyhole (16).

\section{Statistical analysis}

Statistical Package for the Social Sciences (SPSS) version 20 was used for data analysis. Descriptive statistics were summarized by frequency and proportions. Continuous data were summarized as median along with interquartile range (IQR) values.

\section{Results}

A total of 282 archived rectal swabs collected from hospitalized children, 139 (49\%) from KCMC, 74 (26 \%) from Mawenzi and 69 (25\%) from Pasua were included in the study. The majority of the children were males (56.7\%). Most of participants (39\%) were aged between 3 and 11 months with the median age of 18 months (IQR8.0-44.25). Escherichia coli were isolated from all study children. All isolates were susceptible to amikacin and meropenem. However, AMR was observed on ampicillin and Trimethoprim with $83.3 \%$ and $75.9 \%$ of the isolates been resistant. Resistant to ceftazidime was observed on $29.4 \%$ of the isolates; ciprofloxacin $27.7 \%$; gentamicin $21.3 \%$; pipercillin-tazobactam $8.2 \%$; amoxicillin/clavunic 24.5\%; ceftriaxone $30.1 \%$ and cefuroxime $32.6 \%$ (Table 1 )

Table 1 : Number (\%) of E.coli isolates resistant to different antibiotics

Out of $282 \mathrm{E}$. coli isolates in this study $37(13.1 \%)$ were found to be ESBL producers. All $37(100 \%) \mathrm{ESBL}$ producers were resistant to ampicillin and cefuroxime while varying degree of AMR was shown by 28 (75\%) on ciprofloxacin, $33(89.2 \%)$ on trimethoprim $20(54.1 \%)$ on gentamicin $31(83.8 \%)$ on ceftazidime 
and $36(97.3 \%)$ on ceftriaxone. All ESBL producers were $100 \%$ sensitive to amikacin and meropenem (Table 2).

Table 2: Antimicrobial susceptibility to commonly used antimicrobial classes among ESBL producing E. coli isolates $(\mathrm{N}=37)$

Among 245 non ESBL producers, high resistance was observed on ampicillin 198(80.8\%) and trimethoprim 181(73.9\%).All non ESBL producers were 100\% sensitive to amikacin and meropenem (Table 3).

Table 3: Antimicrobial susceptibility to commonly used antimicrobial classes among non Extended spectrum beta lactamase producing Escherichia coli. coli isolates $(\mathrm{N}=245)$

\section{Discussion}

All E. coli isolates that were analyzed demonstrated high rates of resistance to commonly used classes of antimicrobial. Similar observations have been reported by previous studies in Mwanza $(9,15)$.

Escherichia coli showed a high rate of resistance to ampicillin. This high rate of resistance of $E$. coli to ampicillin could be contributed by its easy availability in the pharmacy at inexpensive prices affordable by everyone and is highly prescribed by clinicians since is a first line antibiotic as per National standard antibiotic guidelines (17) sometimes without doing culture and antimicrobial susceptibility testing leading to its overuse and misuse $(18,19)$.

All Escherichia. coli isolates were susceptible to amikacin and meropenem similar to previous reports $(20,7,21)$. The low rates of resistance to amikacin and meropenem could be explained by the rare use of these antimicrobial since are second line according to National standard antibiotic guidelines (17)

The prevalence of ESBL producing E. coli in this study was higher as compared to reports on globally isolated E. coli (21). However, it was lower as compared to the findings in hospitalized children in Dar es Salaam (22) and in Bahran (20) and Rwanda (23). The easily accessible and the habit of using third generation cephalosporin which is a broad spectrum without culture and antibiotic susceptibility testing may influence on the overuse of the drugs. AMR among ESBL producing bacteria to cephalosporin has been reported to be associated with over exposure to this class of drugs $(24,25)$.

The high rate of antimicrobial resistance was observed on cephalosporins followed by penicillin, sulphonamide and fluoroquinolones among ESBL producing E. coli. These are easily available and commonly prescribed by clinicians, hence creating the pressure of excessive antimicrobial use in Moshi. Similarly, a study in Mwanza, Tanzania reported an increase in the rate of AMR to third generation cephalosporins overtime among commonly prescribed antibiotics (9). The increases of pressure in antimicrobial use have been reported to promote development of AMR amongst different bacterial species and strains through horizontal gene transfer and thus contribute to the rapid dispersal in the community (10). 


\section{Limitations}

- Use of archived sample

\section{Declarations}

\section{Ethical considerations}

The study was carried out in accordance with existing ethical guidelines. Ethical clearance was obtained from the Kilimanjaro Christian Medical University College Research Ethics Committee with certificate number 2030.

\section{Consent to publish}

Not applicable

\section{Availability of data and materials}

Materials and data available in this document can be freely used by any scientist wishing to use them for non-commercial purposes as reference materials for further study.

\section{Competing interest}

I declare that there is no any competing interest as far as this study is concern

\section{Funding}

Not applicable

\section{Authors' contributions}

MZELIFA DAUD; this is the main author, did all laboratory procedures.

Balthazar Nyombi; provided advice on how to go about the study including writing research report.

Acknowledgement: We are grateful to the staff of the teaching laboratory of KCMUCo for their participation in this work.

\section{References}

Manyahi J, Matee M.I, Majigo M,Moyo S, Mshana S.E, Lyamuya E.F,2014. Predominance of multi-drug resistant bacterial pathogens causing surgical site infections in Muhimbili national hospital, Tanzania. BMC Res Notes. 7:500

Neil W, Jane F, T, David M, 2011. Multiresistant Gram negative bacteria: the role of high-risk clones in the dissemination of antibiotic resistance. FEMs Microbiology Rev. 35 (5): 736-755. 
Magiorakos A.P, Srinivasan, Carey R.B, Carmeli Y, Faragas M.E, Giske C.G, Harbarth S, Hindler J.F, KahImeter,Olsson-Liljequist B, Paterson D.L, Rice L.B, Stelling J, StruelensM.J, Vatopoulos A, Weber J.T, Monnet D.L, 2011. Multidrug- resistant, extensively drug- resistant and pandrug resistant bacteria: an international expert proposal for interim standard definitions for acquired resistance. Clinical Microbiology infection. 18: 268-281.

Moyo S, Aboud S, Kasubi M, Maselle S.Y, 2010. Bacteria isolated from blood stream infections at a tertiary hospital in Dar es Salaam, Tanzania-antimicrobial resistance of isolates. South African Medical Journal. 100(12):2078-5135.

Lukac P.J, Bonomo R.A, Logan L.K, 2015. Extended -Spectrum Beta-lactamase-producing Enterobacteriaceae in children: old Foe Emerging Threat. Healthcare epidemiology. 60(1):1389.

Souli M, Galani I, Giamarellou H, 2008. Emergence of extensively drug- resistance and pandrug-resistant Gram- negative bacilli in Europe. Europe PMC13(47): 5437-5453.

Alshara M, 2011.Antimicrobial resistant pattern of Escherichia coli strains isolated from pediatric patients in Jordan. Acta Medica, Iranica. 49(5):293-295.

Bryce A, Hay A.D, Lane I.F, Thornton H.V, Wootton M, Costelloe C, 2016. Global prevalence of antibiotic resistance in paediatric urinary tract infections caused by Escherichia coli and association with routine use of antibiotics in primary care: systematic reviewand meta-analysis.

BMJ;352:i939http://dx.doi.org/10.1136/bmj.i939.

Mshana S.E, Kamugisha E, Mirambo M, Chakraborty T, Lyamuya E.F,2009. Prevalence of multiresistant gram-negative organisms in a tertiary hospital in Mwanza, Tanzania. BMC Research Notes. 2:49 DOI: 10.1186/1756-0500-2-49

Hawkey P.M, Jones A.M, 2009. The changing epidemiology of resistance. Journal of Antimicrobial Chemotherapy. 64(1): i3-10. doi: 10.1093/jac/dkp256.

Ahmed M, Moremi N, Mirambo M.M, Hokororo A, Mushi M.F, Seni J, Kamugisha E, Mshana S.E, 2015.Multi-resistant gram negative enteric bacteria causing urinary tract infection among malnourished underfives admitted at a tertiary hospital, northwestern, Tanzania. Italian Journal of Pediatrics. 41:44 doi: 10.1186/s13052-015-0151-5 PMCID: PMC4472394.

Colomb-Cotinat M, Lacoste J, Brun-Buisson C, Jarlier V, Coignard B, Vaux S, 2016. Estimating the morbidity associated with infections due to multidrug-resistant bacteria (MDRB), France. Antimicrobial Resistance infection control. 5:56. Doi: 10.1186/s13756-016-0154-z.

WHO,2018. Global Antimicrobial Surveillance.

Tadesse B.T, Ashley E.A, Ongarello S, Havumaki J, Wijegoonewardena M, Gonzalez I.J, Dittrich S, 2017. Antimicrobial resistance in Africa; a systematic review. BMC Infectious Diseases. 17:616. Doi: 
Moremi N, Claus H, Mshana S.E, 2016. Antimicrobial resistance pattern: a report of microbiological cultures at a tertiary hospital in Tanzania. BMC infectious Disease. 16:756. DOI 10.1186/s12879-0162082-1.

Drieux L, Brossier F, Sougakoff W, Jorlier V, 2008. Phenotypic detection of extended - spectrum betalactamase production in Enterobacteriaceae: review and bench guide. Clinical Microbiology and infection. 14(1):90-103.

Tanzania MOHCDGEC Standard treatment guideline and National essential Medicines list Tanzania Mainland, 2017; pg 419-420.

Bergman M, Nyberg S.T, Huovinen P,Paakkari P, Hakanen A.J,2009. Association between antimicrobial consumption and resistance in Escherichia coli. American Society for Microbiology. 53(3):912-917.

Ayatollahi J,Shahcheraghi S.H, Akhondi R, Soluti S.S, 2013. Antibiotic resistance patterns of Escherichia coli isolated from children in Shahid Sadoughi Hospital of Yazd. Iranian Journal of Pediatric Hematology oncology. 3(2):78-82

Bindayna K.M, Senok A.C, Jamsheer A.E, 2009. Prevalence of extended spectrum beta-lactamase producing enterobacteriaceae in Bahrain. Journal of Infection and Public Health. 2:129-135.

Kehl S.C, Dowzicky M.J, 2015. Global Assessment of Antimicrobial Susceptibility among Gram-Negative Organisms Collected from Pediatric Patients between 2004and 2012: Results from the Tigecycline Evaluation and SurveillanceTrial. Journal of Clinical Microbiology. 53:1286-1293.

Tellevik MG, Blomberg B, Kommedal Ø, Maselle SY, Langeland N, Moyo SJ, 2016. High Prevalence of Faecal Carriage of ESBL-Producing Enterobacteriaceae among Children in Dar es Salaam, Tanzania. PLOS ONE. 11(12): e0168024. https://doi.org/10.1371/journal.pone.0168024.

Ntirenganya C, Manzi D, Mavunyi C.M, Ogbuaga O, 2015. High prevalence antimicrobial resistance among common bacterial isolates in a tertiary Healthcare Facility in Rwanda. American Journal of Tropical Medicine and Hygiene. 94(4):865-870.

Zerr D.M, Miles-Jay A, Kronman M.P, Zhou C, Adler A.L, Haaland W,Weissman S.J, Elward A, Newland J.G, Zaoutis T, Qin X, 2016. Previous antibiotic exposure increases risk of infection with extended-spectrum beta lactamase and AmpC-producing Escherichia coli and Klebsiella pneumoniae in pediatric patients. Antimicrobial Agents Chemotherapy.60:4237-4243. doi:10.1128/AAC.00187-16.

Dohmen W, Dorado-García A, Bonten M.J.M, Wagenaar J.A, Mevius D, Heederik D.J.J, 2017. Risk factors for ESBL-producing Escherichia coli on pig farms: A longitudinal study in the context of reduced use of antimicrobials. PloS ONE. 12(3):e0174094. https://doi.org/10.1371/journal.pone.0174094. 


\section{Supplementary Files}

This is a list of supplementary files associated with this preprint. Click to download.

- supplement1.docx 